\title{
An Automated Optical Inspection system for a tube inner circumference state identification
}

\author{
Chung-Wen Hung*, Jhen-Gu Jiang, Hsien-Huang P. Wu, Wei-Lung Mao \\ Department of Electrical Engineering, National Yunlin University of Science \& Technology, 123 University Road, \\ Section 3,Douliou Yunlin 64002, Taiwan, R.O.C
}

\author{
E-mail: wenhung@yuntech.edu.tw \\ www.yuntech.edu.tw
}

\begin{abstract}
An automated optical inspection, AOI, system for a chopstick tube inner circumference state identification is proposed. This AOI system is installed after the material feed equipment to screen for cost reduction. The tube status identification is based on the proposed machine vision software, and implemented with EmguCV library. The algorithms of image processing are used to sort the material into five groups. In this paper, the algorithm will be detailed, and the experimental results will show the proposed AOI system is workable.
\end{abstract}

Keywords: Machine Vision, AOI, EmguCV, Tube inner circumference.

\section{Introduction}

As AOI technology advances, it is adopted in more and more production lines, but due to cost, not for traditional industry factories, such as chopstick factories. An expensive process for chopsticks is the chopstick defect detecting, and laborers check the good quality only in the final phase.. However, most of the defective products are caused by the original flawed material. Only rely on the naked eye to determine the defects, it is too difficult and waste time. In the other hand, the optical detection methods will not damage the surface of the tube. An AOI system for chopstick material is proposed in this paper. Machine vision technology is used to screen out the tube for flaws before they are processed. In order to have the recovery of defective materials, the defects are sorted into four types: "Large", "Small", "Deformed" and "Empty".

In machine vision, the quality of object imaging can be a critical part. The complexity and accuracy of the algorithm are related to it. Therefore, the Charge-coupled Device, CCD, and telecentric lens are used to enhance the accuracy of the machine vision system. In order to prevent the influence from the environment light, a stable light source is needed [1], and a circular light source is used to ensure uniform illumination of each tube. The proposed system is based on the machine vision software library, EmguCV[2], to quickly confirm a tube was imaged clearly and in compliance with the imaging requirements of the defect inspection.

\section{Related researching}

\subsection{Region of Interest, $\mathrm{ROI}$}

The purpose of ROI is to separate the images captured by the CCD in a particular area by filters, binarization, image cuts, etc. Moreover, it is used to reduce the computational resources required in image processing [3], and it can also reduce the error in the operation of subsequent algorithms with the prior threshold design. 


\subsection{Edge detection}

After the image filtering, grayscale, binarization, the black-andwhite boundary point in the ROI will been detected and marked, and the marked line segment is the outline which is the peripheral line of the tube. In many algorithms, Canny detector[4] is more effective in the edge extraction, so in this paper, Canny detector is used to extract the edge of the tube.

\subsection{Component Labeling}

When edges or binarization of an image is detected, labeling is used to cluster the pixels connected to the same area into the same object. Its purpose is to isolate the noise in some images[5] or the non-interesting content that can not be completely removed in the previous step. These noise and non-interesting content can be ruled out by simple threshold judgment, and then the most complete image information for subsequent feature extraction is got.

\section{The development library: EmguCV}

There are many software packages developed for AOI, and they are also popular. Such as Halcon, an image processing software from Germany, claims the most effective computer vision software in Europe and Japan. However, due to cost issue, this paper developed the system based on open source libraries. EmguCV is an open source image processing development kit that includes the many relevant libraries required for many applications such as image processing, machine learning and computer vision. Users can choose according to their own needs different programming languages such as $\mathrm{C} \#, \mathrm{VB}, \mathrm{C}++$.and so on. EmguCV solved the lack of OpenCV Graphical User Interface, GUI, and is suitable for more application development.

\section{Hardware specifications and configuration}

Table 1 shows the specifications of the CCD and lens used in the system proposed this paper. The CCD with a telecentric lens can effectively reduce the image deformation which caused by the normal lens [1]. In order to average the light on each tube and minimize the shadow [6,7], the light source used in this system is a circular parallel ring light. Figure 1 is the configuration for camera, lens, light and tubes used in this paper. The relative distance between the lens and the tube depends on the working distance of the lens itself.

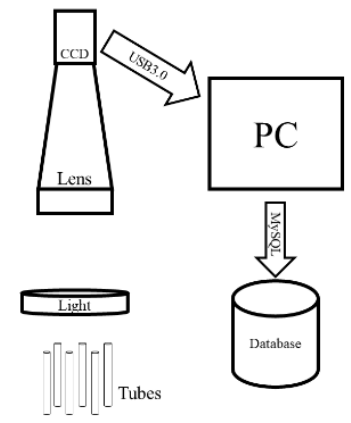

Figure.1 Schematic diagram of the AOI
Table 1. Specification of the CCD and lens

\begin{tabular}{lc}
\hline & Camera \\
\hline Interface & USB 3.0 \\
Sensor type & CMOS \\
Shutter & Rolling shutter \\
Resolution & Lens \\
\hline & 0.184 \\
\hline Magnification & $132.9 \mathrm{~mm}$ \\
Working distance & Computer \\
\hline & Windows 7 \\
\hline OS & I7-5500 x64 \\
CPU & Visual Studio 2017 \\
Development tools &
\end{tabular}

5. Algorithm and flow chart

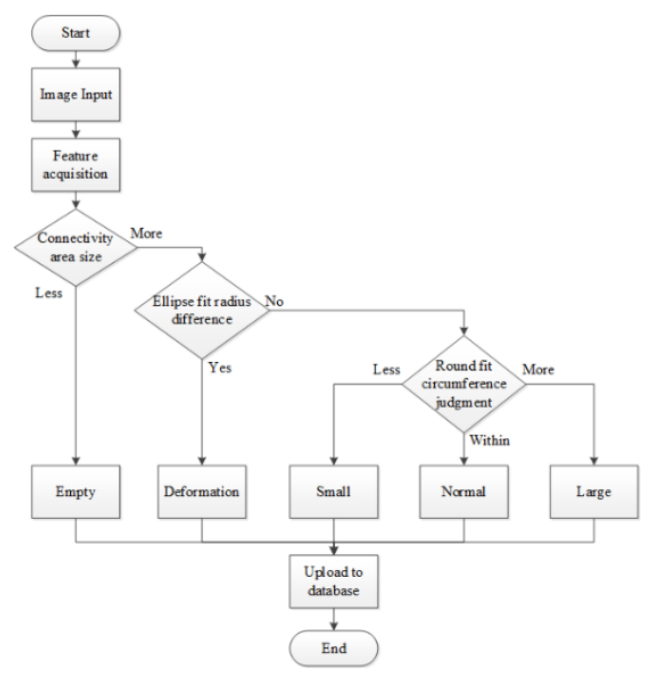

Figure.2 A flowchart of the system

The system flow chart is shown in Figure 2. The main purpose of the system is the inner circumference measurement of the of the chopstick tube. There is no any image filter adopted in the preprocessing stage in this proposed system. Because the maintenance of the image original information is most important, and filters may remove some critical information. The AOI system sorts the defect materials into five modes: one empty situation, and four defect types. The inspection algorithms are detailed in following:

\subsection{Empty}

By connecting the size of the region, the system could determine whether the tube on the fixture. If the connected region is bigger than the setting threshold, the system will indicate no tube on the fixture, otherwise. The threshold setting is based on analysis of the inner area of pre-shot photographs. 


\subsection{Deformation tube}

The ellipse fitting algorithm is used to fit an under testing tube to the most suitable ellipse [8]. The difference between the two radii of the fitting ellipse is used to determine whether the tube has a deformation situation. When the difference is bigger than the setting threshold (about 8 pixels), the tube will be denoted as "deformation".

\subsection{Iron filing elimination algorithm}

Except empty and deformation, iron filing effect has to be eliminated. The iron filings effect is caused in the process or factory environment, and these iron filings have significant influences on the accuracy in the precise identification. The iron filing elimination algorithm is proposed to eliminate this effect. The Inscribed and circumcircled circles are estimated [8]. When the circumference difference of the two circles is big, the circle fitting is used to estimate the tube inner circumference. Contrary in small difference, what means minor iron filing effect, the circumference is integrated from pixels directly.

\section{Experimental results}

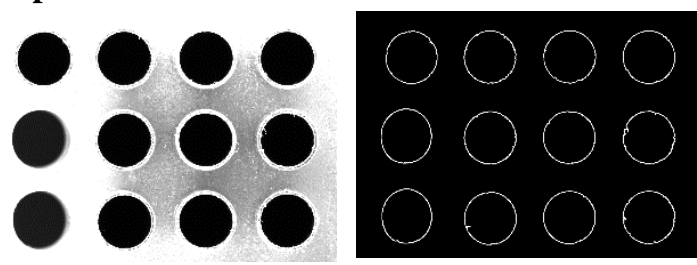

Figure.3 Edge detection

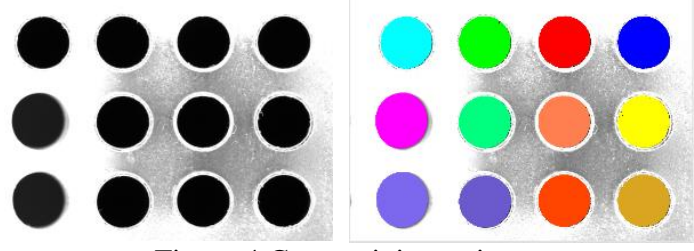

Figure.4 Connectivity region

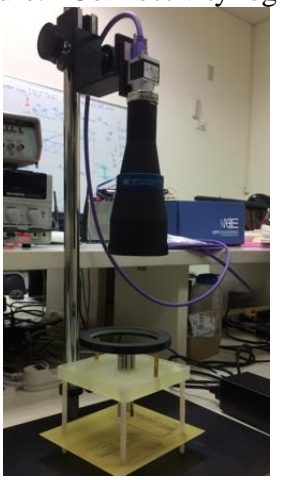

Figure.5 Photo of the AOI system

\subsection{Experimental hardware system}

The block diagram and photo of the proposed system are shown in Figure 1 and 5. The results of the pre-processing are present in Figure 3 and Figure 4, what are Edge detection and connectivity region functions.

\subsection{Circumference Threshold}

The circumference threshold is a key parameter to sort the tube into "Normal", "Large" and "Small" types when not empty and deformation. As Figure 6, the tubes of three types provided by the factory are measured, then threshold could be decided.

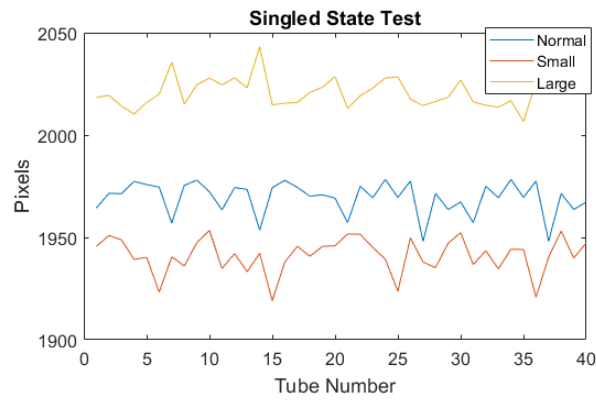

Figure.6 Single state comparison graph

\subsection{Mix state test results}

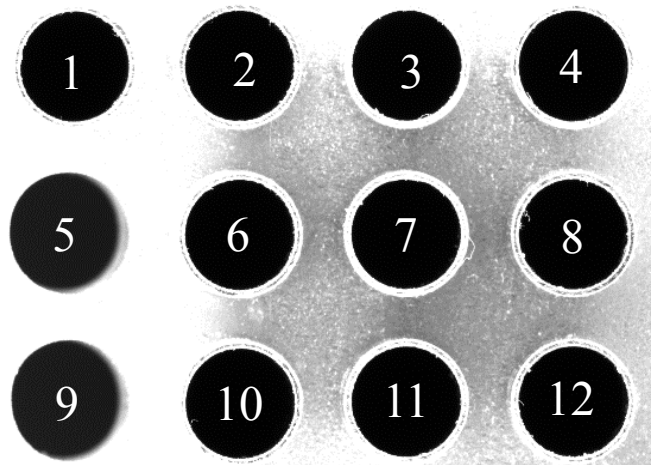

Figure.7 Serial Numbering
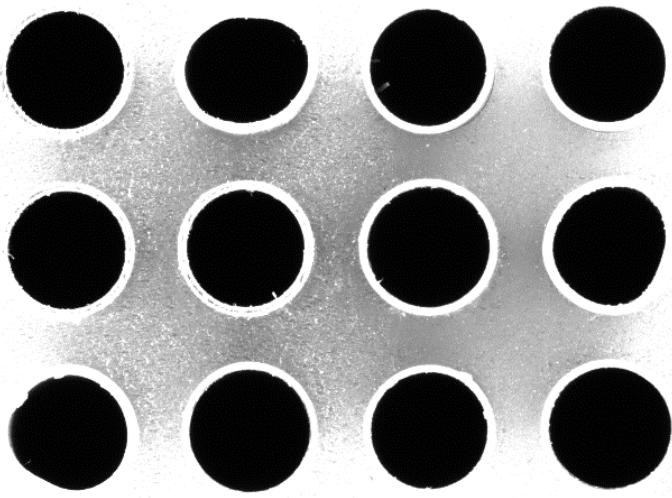

Figure. 8 Mixed test 1 


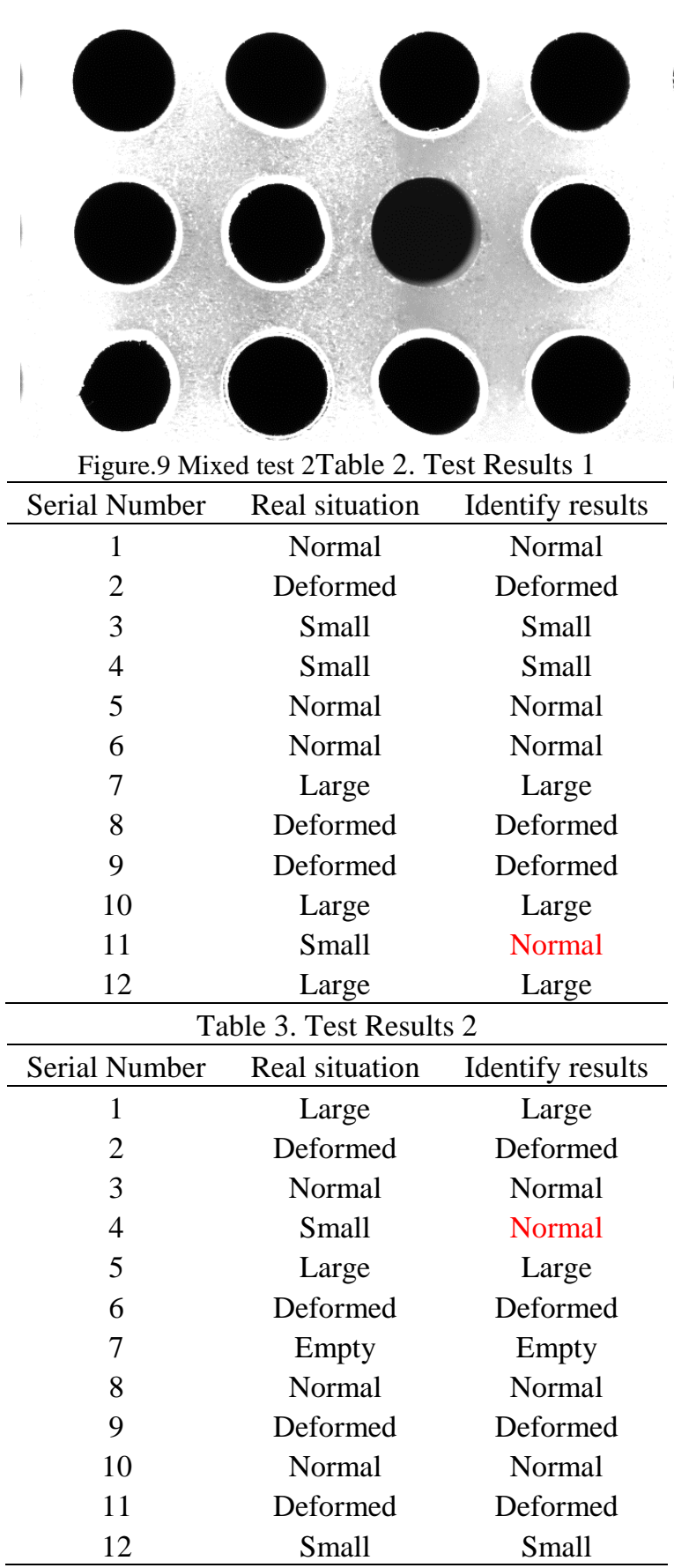

Figure 7 shows the serial number of tubes. The Figure 8 and 9 are the sampling photo, and the Tables 2 and 3 are the inspection results. There is only one error in every test, and the recognition rate of the algorithm reaches $91.67 \%$.

\section{Conclusion}

An AOI system for a chopstick tube inner circumference state identification is proposed, in this paper. The system can effectively identify a variety of different types of the tube by comparing various parameters and threshold design. The experimental result indicates the $91.67 \%$ of the recognition rate, and the system can be used in automated production lines and effectively reduce the production loss.

\section{Acknowledgment}

This work is partially supported by the Ministry of Science and Technology, ROC, under contract No. MOST 106-2221-E-224025, and 106-2218-E150-001.

\section{References}

1. H. Liao, Z. Chen and X. Zhang, "Calibration of Camera with Small FOV and DOF Telecentric Lens", Preceding of 2013 IEEE International Conference on Robotics and Biomimetics, Shenzhen, China, 2013.

2. EmguCV.org Available at www.emgu.com/wiki fourth November 2017

3. W. C. Tham, S.I. Woolley, S. Cribbs and D. Anderson, "Diagnostically lossless compression of pipeline inspection data", Proceedings of Data Compression Conference 2002, Snowbird, UT, USA, 2002

4. J. Canny, "A computational approach to edge detection", IEEE Transactions on Pattern Analysis and Machine Intelligence. Vol. PAMI-8, Iss. 6, Nov. 1986

5. J. Song, Z. Zhao, Q. Zeng and Y. Wei, "An algorithm for eliminating the isolated regions based on connected area in image classification" Preceding of IGARSS 2004. 2004 IEEE International Geoscience and Remote Sensing Symposium, Anchorage, AK, USA, 2004

6. K. W. Khawaja, A. A. Maciejewski, D. Tretter, and C. A. Bouman, "Camera and Light Placement for Automated Assembly Inspection" Preceding of Robotics and Automation, 1996. Proceedings of IEEE International Conference on Robotics and Automation, Minneapolis, MN, USA, USA, 1996

7. Mónica Valenzuela-Delgado Wendy Flores-Fuentes, Miguel E. Bravo-Zanoguera, Alejandro S. Ortiz-Pérez, Daniel Hernandez-Balbuena, Moises Rivas-López, Oleg Sergiyenko, "Felix F. Gonzalez-Navarro, Machine vision system to measuring the velocity field in a fluid by Particle Image Velocimetry: Special Case of Magnetohydrodynamics" 2017 IEEE 26th International Symposium on Industrial Electronics (ISIE), Edinburgh, UK, 2017

8. Truc Le and Ye Duan, "CIRCLE DETECTION ON IMAGES BY LINE SEGMENT AND CIRCLE COMPLETENESS", 2016 IEEE International Conference on Image Processing, Phoenix, AZ, USA, 2016 